\title{
Abordagem da automedicação contra COVID-19 pelo Médico de Família e Comunidade
}

\author{
Approach to self-medication for COVID-19 by the family and community physician \\ Enfoque de la automedicación contra COVID-19 por el Médico de Familia y Comunidad
}

\author{
Waldemir de Albuquerque Costa ${ }^{\oplus}$, Natalia de Campos Carvalho ${ }^{\circledR} \odot$, Pedro Alexandre Barreto Coelho ${ }^{3} \odot$
}

'Secretaria de Saúde do Distrito Federal - Brasília (DF), Brasil.

2Grupo Especial de Supervisão do Programa Mais Médicos no Amazonas - Manaus (AM), Brasil.

${ }^{3}$ Secretaria de Saúde do Distrito Federal - Brasília (DF), Brasil.

\section{Resumo}

Introdução: A automedicação para COVID-19 é considerada um problema emergente e que reflete o momento de infodemia e de crescimento da doença no Brasil. Nesse sentido, a Atenção Primária à Saúde, em que ocorre a maior parte do acesso dos casos leves e moderados de COVID-19, tem-se mostrado um lugar privilegiado para a abordagem de pacientes em uso irracional desses medicamentos. Objetivo: Discutir questões sobre a automedicação voltada para a COVID-19, abordando particularidades do processo de trabalho do médico de família e comunidade (MFC) e as perspectivas para esses profissionais num cenário de grandes tensões político-sanitárias. Métodos: Trata-se de um ensaio teórico que se baseia nas premissas do uso racional de medicamentos (URM) e da medicina baseada em evidências para sintetizar uma proposta de atuação de MFC à luz dos principais documentos e normativas produzidas sobre o tratamento da COVID-19 no país. Resultados: Por meio do referencial teórico, são apresentadas duas estratégias principais para a abordagem dos casos de automedicação para COVID-19: uma do ponto de vista individual, que envolve a desprescrição desses medicamentos mediante um entendimento acordado entre o MFC e o paciente; e uma de cunho sistêmico, ligada ao combate à venda irregular de medicamentos voltados para a COVID-19 no território de atuação desse profissional. Conclusões: As disputas políticas em torno da pandemia ainda continuam vivas e devem persistir por longa data no Brasil. Cabe aos profissionais de saúde e à sociedade como um todo defender o URM e combater a utilização de medicamentos desnecessários, inefetivos, inseguros ou potencialmente inadequados no contexto da COVID-19, protegendo assim a vida e o bom uso dos recursos da população.

Palavras-chave: Automedicação. Infecções por coronavírus. Atenção primária à saúde. Medicina de família e comunidade.

Como citar: Costa WA, Carvalho NC, Coelho PAB. Abordagem da automedicação contra COVID-19 pelo Médico de Família e Comunidade. Rev Bras Med Fam Comunidade. 2021;16(43):2880. https://doi.org/10.5712/rbmfc16(43)2880

\author{
Autor correspondente: \\ Waldemir de Albuquerque Costa \\ E-mail: doutorwal@gmail.com \\ Fonte de financiamento: \\ não se aplica. \\ Parecer CEP: \\ não se aplica \\ Procedência: \\ não encomendado. \\ Avaliação por pares: \\ externa. \\ Recebido em: 25/01/2021. \\ Aprovado em: 31/05/2021. \\ Editor Associado: \\ Leandro David Wenceslau
}




\begin{abstract}
Introduction: Self-medication for COVID-19 is considered an emerging problem, and it reflects an infodemic and growth of the disease in Brazil. Accordingly, primary health care (PHC), where most of the access of mild and moderate cases of COVID-19 occurs, has been shown to be a special place for approaching patients using these drugs irrationally. Objective: To discuss questions about self-medication for COVID-19, addressing particularities of the work of the family and community physician and the perspectives for these professionals in a scenario of great political-health tensions. Methods: This was a theoretical study based on the premises of the rational use of medicines (RUM) and evidence-based medicine to put forward a proposal for the performance of family physicians in the light of the main documents and regulations produced on the treatment of COVID-19 in the country. Results: Through the theoretical framework, two main strategies were presented for addressing cases of self-medication for COVID-19: one from an individual point of view, which involves the prescription of these drugs through an understanding agreed between the family physician and the patient; and one of a systemic nature, linked to the fight against the illegal sale of medicines for treating COVID-19 in the area where this professional works. Conclusions: Political disputes over the pandemic still go on and should persist for a long time in Brazil. It is up to health professionals and society as a whole to defend RUM and combat the use of unnecessary, ineffective, unsafe or potentially inappropriate medicines in the treatment of COVID-19, thus safe-guarding life and good use of the population's resources.
\end{abstract}

Keywords: Self-medication. Coronavirus infections. Primary health care. Family practice.

\title{
Resumen
}

Introducción: La automedicación para COVID-19 se considera un problema emergente y refleja el momento de la infodemia y el crecimiento de la enfermedad en Brasil. En este sentido, la Atención Primaria de Salud (APS), donde ocurre la mayor parte del acceso de casos leves y moderados de COVID-19, ha demostrado ser un lugar privilegiado para acercarse a pacientes en uso irracional de estos fármacos. Objetivo: Discutir cuestiones sobre automedicación dirigidas al COVID-19, abordando las particularidades del proceso de trabajo del médico de familia y comunidad (MFC) y las perspectivas de estos profesionales en un escenario de grandes tensiones político-sanitarias. Métodos: Se trata de un ensayo teórico que se basa en las premisas del uso racional de medicamentos (URM) y la medicina basada en evidencias (MBE) para sintetizar una propuesta para el desempeño de los MFC's a la luz de los principales documentos y normativas producidas em el tratamiento de COVID-19 en el país. Resultados: A través del marco teórico, se presentan dos estrategias principales para el abordaje de los casos de automedicación por COVID-19: una desde un punto de vista individual, que involucra la prescripción de estos fármacos a través de un entendimiento consensuado entre el MFC y el paciente; y una de carácter sistémico, vinculado a la lucha contra la venta irregular de medicamentos dirigidos al COVID-19 en el territorio donde trabaja este profesional. Conclusiones: Las disputas políticas sobre la pandemia aún están vivas y deberían persistir durante mucho tiempo en Brasil. Corresponde a los profesionales de la salud y a la sociedad en su conjunto defender la URM y combatir el uso de medicamentos innecesarios, ineficaces, inseguros o potencialmente inapropiados en el contexto del COVID-19, protegiendo así la vida y el buen uso de los recursos de la población.

Palabras clave: Automedicación. Infecciones por coronavirus. Atención primaria de salud. Medicina familiar y comunitaria.

\section{INTRODUÇÃO}

A pandemia do novo coronavírus (SARS-CoV-2), causador da COVID-19, tem-se alastrado pelo mundo e avançado por todos os estados brasileiros, com destaque para os períodos entre junho e agosto de 2020 e entre março e junho de 2021. Até 27 de julho de 2021, o Brasil já alcançava a marca de 19.749.073 casos e 551.835 mortes pela doença. ${ }^{1}$ A relevante letalidade, a facilidade de contágio e a falta de medicamentos específicos contra a COVID-19 provocaram grande apreensão na população brasileira, bem como expuseram a fragilidade das redes de saúde de estados e municípios, trazendo à tona limitações importantes no acesso aos serviços. ${ }^{2}$ Nesse contexto, medos e incertezas têm intensificado o hábito de automedicação - uso de medicamentos sem prescrição, orientação ou acompanhamento do médico ou dentista, seja por fármacos de venda livre, seja por comércio irregular. ${ }^{3}$

No primeiro semestre de 2020 cresceu consideravelmente a divulgação de fake news e informações preliminares de pesquisas científicas em redes sociais e na mídia acerca de fármacos já existentes no mercado que apresentariam potencial na prevenção e/ou tratamento da COVID-19.4 Muitos desses medicamentos, promissores em análises in vitro, mostraram-se ineficazes para a doença quando submetidos a estudos clínicos em humanos ${ }^{5}$ — contudo, a visibilidade trazida a tais terapias despertou uma 
busca desenfreada por farmácias jamais vista no país. ${ }^{6}$ Segundo estudo do Conselho Federal de Farmácia (CFF), ${ }^{7}$ a venda de ivermectina no Brasil apresentou crescimento de $557 \%$ entre 2019 e 2020 , com mais de 8 milhões de caixas vendidas somente no mês de junho de 2020. A procura excessiva por hidroxicloroquina levou ao desabastecimento de inúmeras farmácias, comprometendo o tratamento de pacientes portadores de doenças reumáticas, e o comércio ilícito de azitromicina aumentou consideravelmente tanto em feiras livres quanto em farmácias de pequeno porte, sem a exigência de receituário especial, ou mesmo em drogarias clandestinas. ${ }^{3,7}$ Por outro lado, o desequilíbrio da oferta/demanda desses medicamentos resultou em aumento significativo dos preços, prejudicando o acesso dos demais usuários. ${ }^{8}$

Para tentar conter a explosão de vendas de tais medicamentos, a Agência Nacional de Vigilância Sanitária (ANVISA) incluiu em abril de 2020 a cloroquina e a hidroxicloroquina na lista de substâncias sujeitas à Receita de Controle Especial. ${ }^{9}$ Além disso, a aprovação pelo Conselho Federal de Medicina $(C F M)^{10} \mathrm{e}$ pelo Ministério da Saúde (MS) ${ }^{11}$ da sua prescrição para casos de COVID-19 foi condicionada à assinatura de termo de ciência e consentimento pelo usuário, o que restringiu ainda mais a sua comercialização. Com isso, a compra dos medicamentos foi migrando sucessivamente para os vermífugos, especialmente a ivermectina, de maior acessibilidade e menor custo., ${ }^{3,7}$ Diante dessa movimentação, a ANVISA passou a exigir a partir de julho de 2020 receituário especial também para a ivermectina e a nitazoxanida, porém terminou revogando a decisão três meses após sua implantação. ${ }^{12,13}$

A polêmica em torno desses medicamentos tornou-se ainda mais emblemática durante o colapso do sistema de saúde de Manaus em janeiro de 2021, após o então ministro da saúde e o presidente da república atribuírem boa parte das hospitalizações e mortes por COVID-19 à falta de "tratamento precoce" na cidade. ${ }^{14}$ As falas foram contrapostas pelo médico coordenador da unidade de terapia intensiva do Hospital Universitário Getúlio Vargas (HUGV), que afirmou que a automedicação generalizada da população de Manaus não evitou o agravamento dos casos, a superlotação do serviço e o aumento no número de óbitos pela doença. ${ }^{15}$ Outros municípios foram além e passaram a institucionalizar tais práticas nas prefeituras, realizando a distribuição de "kits COVID-19" (combinações de azitromicina, ivermectina e outras substâncias) para a população, muitas vezes de forma pulverizada e sem supervisão médica. ${ }^{16}$ Em levantamento realizado com todos os dez municípios brasileiros com mais de 100 mil habitantes que distribuíram oficialmente esses kits, nove deles registraram taxas de mortalidade por COVID-19 mais altas que a média de seus respectivos estados. ${ }^{17}$

Nesse sentido, a automedicação para COVID-19, além de não oferecer proteções adicionais contra a doença e apresentar riscos por interações e efeitos adversos dos fármacos, tem gerado falsa sensação de segurança e levado muitos usuários a abandonarem medidas de higiene e distanciamento social. 2,18

Diante desse cenário, o uso irracional de medicamentos no contexto da COVID-19 tornou-se um problema emergente ${ }^{3}$ e que precisa ser abordado com cautela sobretudo pela Atenção Primária à Saúde (APS), em que ocorre a maior parte do acesso dos casos leves e moderados da doença. ${ }^{19}$ Assim, este ensaio teórico tem por objetivo discutir questões relativas à descontinuidade do uso por automedicação para COVID-19 e sua vigilância territorial, abordando particularidades do processo de trabalho do médico de família e comunidade (MFC) e as perspectivas para esses profissionais em um cenário de grandes tensões político-sanitárias.

\section{MÉTODO}

Este trabalho baseia-se nas premissas do uso racional de medicamentos (URM $)^{20}$ e da medicina baseada em evidências (MBE) ${ }^{21}$ para sintetizar uma proposta de atuação de MFC à luz dos principais 
documentos e normativas produzidas sobre o tratamento da COVID-19 no país - incluindo as publicações das sociedades brasileiras de Medicina de Família e Comunidade (SBMFC), Infectologia (SBI) e Pneumologia e Tisiologia (SBPT), das associações de Medicina Intensiva Brasileira (AMIB) e Médica Brasileira (AMB), do CFM e MS. ${ }^{10,11,22-25}$ O URM, entendido como a administração de medicamentos apropriados à condição clínica, em doses e períodos de tempo adequados ao menor custo possível para o paciente/comunidade, ${ }^{19}$ tem como um de seus pilares a MBE - a utilização integrada das melhores evidências disponíveis na literatura com a experiência clínica do profissional e as preferências dos usuários. ${ }^{20}$ Neste esforço teórico, reconhece-se a ausência de comprovação científica da eficácia de terapias medicamentosas (profiláticas, precoces ou tardias) para a COVID-19 até o presente momento, o que exige dos profissionais uma avaliação caso a caso. ${ }^{10,11,22-25}$ A ausência de evidências fortes (ou a existência somente de evidências indiretas e preliminares de baixo nível de certeza), ${ }^{26}$ dessa forma, impede que sejam feitas recomendações generalizadas de determinados fármacos para a população, como uma espécie de kit, e coloca a automedicação para COVID-19 e o URM em polos radicalmente opostos. ${ }^{27}$ Por fim, dois documentos ${ }^{10,11}$ versam sobre a autonomia profissional dos médicos para a prescrição ou não desses medicamentos em casos de COVID-19, o que chancela a desprescrição de tratamentos ineficazes quando em decisão compartilhada com os usuários.

Por meio dessas diretivas, serão apresentadas duas estratégias principais para a abordagem dos casos de automedicação para COVID-19: uma do ponto de vista individual, que envolve a desprescrição ${ }^{28}$ de tais medicamentos mediante um entendimento acordado entre o MFC e o paciente; e uma de cunho sistêmico, ligada ao combate à venda irregular de medicamentos ${ }^{29}$ voltados para a COVID-19 no território de atuação desse profissional.

O foco deste trabalho serão os usuários que adquirem (de forma lícita ou não) e consomem medicamentos por conta própria para o tratamento específico da COVID-19 - o que inclui drogas como cloroquina, hidroxicloroquina, azitromicina, ivermectina, nitazoxanida, dexametasona e suplementos vitamínicos e minerais. ${ }^{3}$ Não será incluída neste estudo a abordagem das prescrições realizadas por outros profissionais, dadas as singularidades do encontro profissional-usuário, que requer outros referenciais teóricos. Além disso, não fará parte deste trabalho a análise do tratamento sintomático da doença, como por exemplo o uso de analgésicos e anti-histamínicos, previsto nos documentos acima citados e já utilizado com larga experiência nos casos de síndrome gripal. ${ }^{30}$

\section{Desprescrevendo medicamentos em uso irracional para COVID-19}

A desprescrição de medicamentos é um processo com fundamentação científica e ética que busca modificar a dose, substituir ou eliminar fármacos que não apresentem benefício ou sejam potencialmente danosos para um determinado paciente. ${ }^{28}$ No contexto da pandemia, surge como uma importante estratégia na abordagem de usuários em automedicação para COVID-19. A APS, por meio de atributos como o acesso e a longitudinalidade do cuidado, possui lugar privilegiado na condução de planos de desprescrição, trazendo maior segurança e eficácia para o seu desenvolvimento. ${ }^{31}$ Embora o termo desprescrição guarde relação semântica com o ato prescritivo realizado por outros profissionais, neste trabalho ele será utilizado com o sentido ampliado da descontinuidade do uso por automedicação, aproveitando-se assim seu arcabouço teórico e sua aplicabilidade no contexto da APS. ${ }^{28}$

Usuários em automedicação habitualmente se apresentam na APS durante a busca por testes para COVID-19, por atendimento sintomático, por efeitos adversos dos medicamentos ou mesmo em contato 
ocasional durante visitas domiciliares. ${ }^{3}$ Nessas situações, é importante abordar motivações como o medo da doença, a contaminação de pessoas próximas, a pressão familiar pelo uso desses medicamentos e, se possível, discutir a problemática da infodemia - "excesso de quantidade em detrimento da qualidade das informações sobre COVID-19". ${ }^{32}$

Se por um lado a desprescrição envolve a busca por uma melhor relação risco-benefício-custo, por outro deve ter como princípio as preferências, desejos e expectativas dos pacientes. ${ }^{28} \mathrm{O}$ método clínico centrado na pessoa (MCCP), como elemento do escopo de trabalho do MFC, aponta caminhos que podem facilitar a condução desses casos. Mais do que uma simples sequência, o MCCP traz uma proposta metodológica que busca aproximar as necessidades e expectativas de profissionais e usuários com base em quatro componentes: explorar a saúde, a doença e a experiência da doença; entender a pessoa como um todo; elaborar um plano conjunto de manejo dos problemas; e fortalecer a relação entre a pessoa e o médico. ${ }^{33} \mathrm{Com}$ a compreensão mais adequada do processo de adoecimento do paciente, torna-se possível uma intervenção mais precisa e resolutiva sobre os problemas apresentados.

O MCCP deve ser empregado ainda em contraposição à prática de profissionais que têm rejeitado ou criado repulsão aos usuários em automedicação para COVID-19. Durante a pandemia, tornou-se frequente a polarização entre um suposto "conhecimento científico", apossado por trabalhadores da saúde, e uma "ignorância ideológica" popular que estaria relacionada à manipulação e a fake news sobre o tratamento da doença. ${ }^{2}$ Com isso, tem-se impedido o diálogo com determinados usuários que, por diversas questões, buscam refúgio em um tratamento medicamentoso. Nesses casos, a empatia e a escuta qualificada fazem-se necessárias não só para desprescrever fármacos de forma segura como também para implantar medidas de redução de danos.

Para o desenvolvimento da desprescrição, é necessário observar o contexto do tratamento diante de possíveis interações medicamentosas, seja com outras terapias, seja mesmo dos medicamentos entre si, e efeitos adversos que possam comprometer outras patologias de base, o que pode reforçar a necessidade de sua exclusão. ${ }^{34}$

- $\quad$ Fármacos consumidos em dose única, vistos com mais frequência nos casos da ivermectina e nitazoxanida, dificilmente conseguirão ser contidos a tempo pelos profissionais, restando apenas o seu monitoramento pós-administração. Apesar dos incipientes estudos no tratamento da COVID-19, têm sido

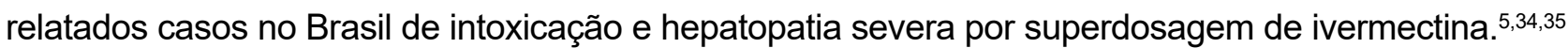

- Drogas com maior duração de uso (de modo profilático ou pós-exposição) podem ser desprescritas em tempo hábil para reduzir danos, sobretudo quando detectadas nos primeiros dias de consumo. Além do tempo de exposição, o número de medicamentos pode ser reduzido ao máximo aceito pelo usuário, excluídos como um todo (supressão) ou eliminados aqueles com maior potencial danoso ("corte seletivo") ${ }^{28}$ — por exemplo, um paciente em uso de um "kit COVID-19" com seis drogas pode refrear grande parte dos riscos à sua saúde ao restringir seu c-onsumo para apenas o suplemento vitamínico. ${ }^{34}$ Vale ressaltar que esse movimento deve ser individualizado, respeitando-se escolhas pessoais e sua correspondência clínica. ${ }^{28}$

A desprescrição pode vir associada ao que chamamos de "demora permitida", quando se desenvolve a observação assistida e não intervencionista dos quadros de menor urgência, ofertando-se, em caso de necessidade, acesso fácil ao seu profissional de referência. ${ }^{36}$ Uma vez que a maioria dos pacientes com COVID-19 experimenta sintomas leves a moderados com resolução espontânea da infecção, é possível ofertar uma desprescrição sóbria e expectante utilizando-se o tempo como instrumento de trabalho (tanto 
terapêutico quanto de esclarecimento diagnóstico). ${ }^{26,27}$ A demora permitida não deve ser confundida com displicência ou omissão - ao contrário, deve ser acompanhada da "observação atenta" ${ }^{6}$ do usuário pelo período de até 14 dias do início dos sintomas, vinculando-o ao serviço e mantendo vigilância sobre os principais sinais de risco. ${ }^{22} \mathrm{Em}$ apoio a esse processo, vale destacar a importância do registro detalhado das informações em prontuário, tomando-se por modelo o Registro de Saúde Orientado por Problemas, como medida de segurança do paciente, de melhoria da comunicação entre os profissionais e de fortalecimento do cuidado longitudinal. ${ }^{37}$

Além da evolução clínica habitual, são frequentes os casos de usuários em automedicação para COVID-19 que buscam atendimento para tratar efeitos adversos dos medicamentos, como exemplo a diarreia após o uso da azitromicina. ${ }^{34}$ Nessas situações, abre-se uma oportunidade de diálogo sobre os riscos potenciais da automedicação e a necessidade de avaliação individualizada na presença de sintomas mais intensos. Entre os efeitos, deve-se dar destaque para a resistência bacteriana que vem sendo potencializada pelo uso irracional da azitromicina, o que pode comprometer o tratamento de doenças como a gonorreia e pneumonias bacterianas. ${ }^{38}$

Por fim, diante do posicionamento tenaz de um usuário quanto ao desejo de manutenção dos medicamentos, devem ser reforçadas as orientações sobre os riscos dos fármacos, deve-se manter o monitoramento do caso e disponibilizar o acesso facilitado em caso de agravamento do quadro clínico. 22,34 A negativa da desprescrição não deve, dessa forma, ser um impeditivo para o cuidado desses pacientes, tornando ainda mais necessária a atenção dos profissionais e o fortalecimento dos canais de diálogo com a população.

\section{Identificando e combatendo o comércio irregular de medicamentos para COVID-19}

Além dos aspectos clínicos individuais, o território de atuação do MFC mostra-se imperativo sobre seu processo de trabalho quando falamos de surtos de automedicação. De modo teórico, a responsabilidade sanitária sobre o território adscrito faz-se presente tanto nas diretrizes do Sistema Único de Saúde (SUS) operacionalizadas pela APS (territorialização/ adscrição de clientela) ${ }^{39}$ quanto nos princípios da medicina de família e comunidade (atuação influenciada pela comunidade/recurso de uma população definida). ${ }^{40}$ Nesse sentido, identificar estabelecimentos que estejam comercializando de forma irregular medicamentos direcionados para a COVID-19 pode ajudar no combate ao seu uso irracional.

A venda fraudulenta de fármacos é uma prática de grave responsabilidade jurídico-penal, porém desenvolvida de forma crônica e disseminada no país. Estão incluídos nessa categoria os atos de contrabando (importação clandestina e descaminho), venda de cargas roubadas, venda de medicações de uso controlado sem a presença de receita especial e o comércio de medicamentos falsificados ou adulterados (com redução, exclusão ou substituição de princípios ativos). ${ }^{41} \mathrm{Em}$ períodos de surto, como o da pandemia de COVID-19, espera-se que o aumento da procura e a limitada capacidade de produção de determinados fármacos no país resulte em crescimento do comércio irregular.

No Brasil, órgãos municipais e estaduais de vigilância sanitária e aANVISA, em atuação compartilhada, são responsáveis pela regulamentação e pela fiscalização de produtos que exigem controle sanitário, seja na inspeção física dos estabelecimentos, seja nos domínios virtuais de atuação nacional. Enquanto à ANVISA compete a coordenação federativa do sistema, cabe às vigilâncias de estados e municípios a atuação local de fiscalização, a elaboração de autos de infrações sanitárias, interdições, apreensões de produtos, entre outros. Esse arranjo institucional, que compõe o chamado Sistema Nacional de Vigilância 
Sanitária (SNVS), rege o acesso seguro aos medicamentos disponibilizados no país - com boa qualidade, eficiência e ausência de contaminantes químicos, biológicos ou físicos. ${ }^{42}$

Contudo, fragilidades no processo de descentralização da vigilância sanitária, como o subfinanciamento e a incipiência do componente municipal do SNVS, têm impactado o desempenho global desse setor e reduzido sua vinculação ao SUS. ${ }^{43}$ Além desses aspectos, a grande extensão territorial do país e a atuação minuciosa e disseminada desses crimes têm dificultado a supervisão do SNVS, situação que se torna ainda mais complexa em um cenário como o da pandemia. Com isso, aumenta a circulação de "medicamentos inseguros", ${ }^{44}$ que podem causar danos irreversíveis à saúde e até a morte de usuários. A coação do comércio irregular de medicamentos, dessa forma, busca não só reduzir o uso indiscriminado de determinadas substâncias como também combater o consumo de medicamentos inseguros no território, evitando a sobreposição de riscos aos pacientes. ${ }^{3}$ Por exemplo, um usuário com sintomas leves de COVID-19, pressionado por familiares para se automedicar com azitromicina, pode acabar adquirindo em uma farmácia irregular um medicamento adulterado, contaminado e fruto de contrabando, agravando seu quadro clínico para além dos riscos já existentes na droga original. Nesse sentido, faz-se ainda mais significativa a atuação dos profissionais da APS pela sua distribuição territorial e proximidade da população. ${ }^{39}$

Após o reconhecimento de um caso de automedicação e a definição das estratégias de desprescrição, é importante questionar o local de compra do medicamento, sobretudo nas situações que exigiriam a presença de receita especial. Essa informação dificilmente é disponibilizada pelo usuário, que teme represálias tanto do local de atendimento quanto da farmácia ou fornecedor de origem. Sendo assim, são fundamentais o diálogo, para tranquilizá-lo sobre a ausência de retaliações no serviço, e a oferta de sigilo ao paciente. Como apontado anteriormente, as motivações para o consumo desses medicamentos são múltiplas e sua abordagem na APS pode ser facilitada quanto maior se mostrar o vínculo com a equipe de referência.

Fármacos como dexametasona, ivermectina, AAS e nitazoxanida possuem venda livre, o que torna sua comercialização facilitada em farmácias e drogarias do país. ${ }^{3}$ Nesses casos é difícil distinguir as situações nas quais ocorre a venda fraudulenta ou mesmo induzida por funcionários. Contudo, é possível aprimorar a comunicação da APS com os farmacêuticos de estabelecimentos da área adscrita para fortalecer a farmacovigilância relacionada a essas drogas, sobretudo em questões como superdosagem, interações medicamentosas e efeitos adversos. ${ }^{45}$

Drogas controladas como a hidroxicloroquina e a azitromicina apresentam situação ainda mais delicada, sendo obtidas nesses casos por intermédio de fraude comercial. ${ }^{3}$ Com isso, destaca-se a importância de dialogar com a população local, informando-a sobre os riscos da compra de medicamentos inseguros, e de tentar, sempre que possível, localizar os estabelecimentos onde eles foram adquiridos. Existem três esferas ${ }^{29}$ para a denúncia de farmácias com venda irregular de medicamentos controlados que podem ser utilizadas pelos profissionais de saúde:

1. Órgãos de vigilância sanitária: entidades municipais, estaduais e ANVISA (por exemplo: central de atendimentos da ANVISA - 0800642 9782; portal eletrônico do Centro de Vigilância Sanitária de São Paulo - www.cvs.saude.sp.gov.br/ouvidoria.asp);

2. Conselhos regionais de farmácia (por exemplo, e-mail para denúncias do Conselho Regional de Farmácia do Rio de Janeiro — denuncia.fiscalizacao@crf-rj.org.br);

3. Ministério Público dos estados, sobretudo as promotorias relacionadas ao setor saúde (por exemplo, Promotoria de Justiça de Defesa da Saúde do Ministério Público do Distrito Federal e Territórios (61) 3343-9472/3343-9440). 
Além desses órgãos, a imprensa local pode ser de grande valia na denúncia de farmácias irregulares e no comércio de rua de medicamentos controlados, como as feiras livres. ${ }^{46}$ Manter o contato com jornais e canais de notícias da região pode ser uma boa estratégia para aumentar a fiscalização sobre esses estabelecimentos e pontos comerciais e coibir, ainda que temporariamente, esse tipo de prática.

Em suma, com essas ações os profissionais da APS podem contribuir para o trabalho global de vigilância em saúde, tanto na identificação de padrões de uso em automedicação entre os usuários e de sua distribuição no território assistido quanto no monitoramento dos casos e de suas repercussões clínicas - valorizando, assim, o conhecimento do território e colaborando para o trabalho de segmentos diversos para além da esfera da saúde.

\section{CONCLUSÕES}

A automedicação para COVID-19 é considerada um problema emergente ${ }^{3}$ e que reflete o momento de infodemia e de crescimento da doença no Brasil. Apesar do avanço na discussão com a sociedade, sobretudo com o apoio de instituições científicas, a venda irregular desses medicamentos mantém-se ativa e disseminada pelo país.

Nesse sentido, a APS tem-se apresentado como lugar privilegiado para a abordagem de tais casos, tanto no aspecto individual das condutas quanto na vigilância do território. O MFC, com as ferramentas de seu escopo de trabalho, mostra-se como um profissional valioso para a condução de processos de desprescrição, redução de danos e vigilância diante do uso indiscriminado desses medicamentos. Contudo, faz-se necessário combater a repulsão de alguns profissionais a tais pacientes, respeitando as motivações individuais e tentando sempre aprimorar o diálogo com a população. Além disso, a identificação e a denúncia dos pontos de comércio irregular desses medicamentos, sobretudo os de venda controlada, na área de atuação dos profissionais é fundamental para reduzir a circulação de medicamentos inseguros entre os usuários.

Por outro lado, a intensificação do debate sobre as vacinas e o início do programa nacional de imunização contra a COVID-19 têm mostrado a importância da valorização da ciência no combate à pandemia - situação que se contrapõe ao uso irracional de medicamentos. A fala de diretores da ANVISA, durante a aprovação emergencial de duas vacinas contra a COVID-19 no Brasil, sobre a "ausência de alternativas terapêuticas" para a doença teve repercussão nacional e impulsionou questionamentos ainda mais incisivos sobre o "tratamento precoce" defendido pelo MS. ${ }^{47}$ Dias após o evento, o então ministro da saúde retratou-se publicamente afirmando não haver incentivado o tratamento precoce, mas sim o "atendimento precoce" de sintomáticos respiratórios, colocando-se contrário à propaganda de qualquer medicamento específico para COVID-19. Por outro lado, o presidente Jair Bolsonaro vem mantendo a defesa dessa terapêutica, incentivando seus seguidores a não desistirem do tratamento precoce. ${ }^{14}$

Nesse sentido, percebe-se que as disputas políticas em torno da pandemia ainda continuam vivas e devem persistir durante longa data no Brasil. Cabe aos profissionais de saúde e à sociedade como um todo defender o URM e combater a utilização de medicamentos desnecessários, inefetivos, inseguros ou potencialmente inadequados no contexto da COVID-19, protegendo assim a vida e o bom uso dos recursos da população.

\section{CONFLITOS DE INTERESSE}

Nada a declarar. 


\section{CONTRIBUIÇÃO DOS AUTORES}

Todos os autores trouxeram contribuições substanciais para a concepção e o delineamento do trabalho e a redação do manuscrito; a revisão crítica; a aprovação da versão final do manuscrito; e a concordância em prestar contas de todos os aspectos do trabalho.

\section{REFERÊNCIAS}

1. Brasil. Ministério da Saúde. Covid-19: painel coronavírus [Internet]. 2021 [acessado em 28 jul. 2021]. Disponível em: https:// covid.saude.gov.br/

2. Do Bú EA, Alexandre MES, Bezerra VAS, Sá-Serafim RCN, Coutinho MPL. Representações e ancoragens sociais do novo coronavírus e do tratamento da COVID-19 por brasileiros. Estud Psicol 2020;37:e200073. https://doi.org/10.1590/1982$0275202037 \mathrm{e} 200073$

3. Brito JCM, Lima WG, Cardoso BG, Simião DC, Amorim JM, Silva CA. Uso irracional de medicamentos e plantas medicinais contra a COVID-19 (SARS-CoV-2): um problema emergente. Brazilian J H Pharm 2020;2(3):37-53. https://doi. org/10.29327/226760.2.3-5

4. Mata ML, Grigoleto MC, Lousada M. Dimensões da competência em informação: reflexões frente aos movimentos de infodemia e desinformação na pandemia da Covid-19. Liinc em Rev 2020;16(2):e5340. https://doi.org/10.18617/liinc. v16i2.5340

5. Pan American Health Organization. Ongoing Living Update of COVID-19 Therapeutic Options: Summary of Evidence. Rapid Review, 14 Jul 2021. $23^{\text {th }}$ ed. [Internet]. 2021 [acessado em 28 jul. 2021]. Disponível em: https://iris.paho.org/bitstream/ handle/10665.2/52719/PAHOIMSEIHCOVID-1921019_eng.pdf?sequence=48\&isAllowed=y

6. Rio Grande do Sul. Conselho Regional de Farmácia. Levantamento mostra como o medo da Covid-19 impactou venda de medicamentos [Internet]. 2020 [acessado em 01 jan. 2021]. Disponível em: https://www.crfrs.org.br/noticias/levantamentomostra-como-o-medo-da-covid-19-impactou-venda-de-medicamentos

7. Conselho Federal de Farmácia. Venda de remédios sem eficácia comprovada contra a Covid dispara [Internet]. 2021 [acessado em 28 jul 2021]. Disponível em: https://www.cff.org.br/noticia. php?id=6197\&titulo=Venda+de+rem\%C3\%A9dios+sem+efic\%C3\%A1 cia+comprovada+contra+a+Covid+dispara

8. Paiva AM, Pinto AWS, Cançado BL, Chequer FMD, Pereira ML, Baldoni AO. Efeito das "promessas terapêuticas" sobre os preços de medicamentos em tempos de pandemia. J Health Biol Sci 2020;8(1):1-5. http://doi.org/10.12662/2317-3076jhbs. v8i1.3407.p1-5.2020

9. Brasil. Ministério da Saúde. Agência Nacional de Vigilância Sanitária. Resolução - RDC n 351, de 20 de março de 2020. Dispõe sobre a atualização do Anexo I (listas de substâncias entorpecentes, psicotrópicas, precursoras e outras sob controle especial) da Portaria SVS/MS n 344, de 12 de maio de 1998, e dá outras providências. Diário Oficial da União, Poder Executivo, Brasília, 20 mar. 2020 [Internet]. 2021 [acessado em 08 nov 2021]. Disponível em: http://www.planalto.gov.br/ ccivil_03/portaria/Resolucao\%20n\%C2\%BA\%20351-ANVISA.htm\#: :text=RESOLU\%C3\%87\%C3\%83O\%20\%2D\%20 RDC\%20N\%C2\%BA\%20351\%2C\%20DE, 1998\%2C\%20e\%20d\%C3\%A1\%20outras\%20provid\%C3\%AAncias

10. Conselho Federal de Medicina. Processo-consulta CFM no 8/2020 - Parecer CFM no 4/2020. Tratamento de pacientes portadores de COVID-19 com cloroquina e hidroxicloroquina. Considerar o uso da cloroquina e hidroxicloroquina, em condições excepcionais, para o tratamento da COVID-19. Brasília, 16 abr. 2020 [Internet]. [acessado em 08 nov 2021]. Disponível em: https://sistemas.cfm.org.br/normas/visualizar/pareceres/BR/2020/4

11. Brasil. Ministério da Saúde. Nota Informativa $n^{\circ}$ 17/2020 - SE/GAB/SE/MS. Orientações do Ministério da Saúde para manuseio medicamentoso precoce de pacientes com diagnóstico da Covid-19. 2020 [Internet]. [acessado em 08 nov 2021]. Disponível em: http://www.consultaesic.cgu.gov.br/busca/dados/Lists/Pedido/Attachments/1527690/RESPOSTA_ RECURSO_1_131580_NOTA\%20INFORMATIVA\%20N\%2017_2020-GAB_SE_MS.pdf

12. Brasil. Ministério da Saúde. Agência Nacional de Vigilância Sanitária. Diretoria Cólegiada. Resolução de Diretoria Colegiada - RDC n० 405, de 22 de julho de 2020. Estabelece as medidas de controle para os medicamentos que contenham substâncias constantes do Anexo I desta Resolução, isoladas ou em associação, em virtude da Emergência de Saúde Pública de Importância Internacional (ESPII) relacionada ao novo Coronavírus (SARS-CoV-2). Diário Oficial da União, Poder Executivo, Brasília, 22 de julho de 2020. [Internet]. [acessado em 08 nov 2021]. Disponível em: https://www.in.gov.br/en/web/dou/-/ resolucao-de-diretoria-colegiada-rdc-n-405-de-22-de-julho-de-2020-268192342

13. Brasil. Ministério da Saúde. Agência Nacional de Vigilância Sanitária. Diretoria Colegiada. Resolução da Diretoria Colegiada - RDC $n^{\circ} 420$, de $1^{\circ}$ de setembro de 2020. Dispõe sobre a atualização do Anexo I da Resolução de Diretoria Colegiada RDC $n^{\circ}$ 405, de 22 de julho de 2020. Diário Oficial da União, Poder Executivo, Brasília, 01 de setembro de 2020. [Internet]. [acessado em 08 nov 2021]. Disponível em: https://www.in.gov.br/en/web/dou/-/resolucao-de-diretoria-colegiada-rdc-n-420de-1-de-setembro-de-2020-275243243

14. Ventura D, Reis R. A linha do tempo da estratégia federal de disseminação da covid-19. In: Direitos na pandemia: mapeamento e análise das normas jurídicas de resposta à Covid-19 no Brasil. 2021;(10):6-31 [Internet]. [acessado em 28 jul 2021]. Disponível em: https://static.poder360.com.br/2021/01/boletim-direitos-na-pandemia.pdf 
15. Olímpio V. Médico de UTI em Manaus rebate acusações de falta de tratamento precoce: "é sacanagem com a gente". Correio Braziliense de 15 de Janeiro de 2021 [Internet]. [acessado em 01 jan 2021]. Disponível em: https://www. correiobraziliense.com.br/brasil/2021/01/4900587-medico-de-uti-em-manaus-rebate-acusacoes-de-falta-de-tratamentoprecoce-e-sacanagem-com-a-gente.html

16. Lobato E. Infodemia, crendice e coronavírus. Vermífugo "até melhor que a cloroquina" vira estrela de kit-Covid de prefeitos e ganha aval de Bolsonaro. Folha de São Paulo de 12 de junho de 2020 [Internet]. [acessado em 01 jan 2021 ]. Disponível em: https://piaui.folha.uol.com.br/infodemia-crendice-e-coronavirus/?fbclid=IwAR0vErt2CGXav3Y5Qe7lgK2azjwoKrveumz C48OVqg6rrZnjNIhPRAEA0Nc

17. Schelp D. Em dez cidades com "kit covid", nove tiveram taxa de mortalidade mais alta. UOL Notícias de 21 de janeiro de 2021 [Internet]. [acessado em 22 jan 2021]. Disponível em: https://noticias.uol.com.br/colunas/diogo-schelp/2021/01/21/ nove-em-dez-cidades-com-kit-covid-tiveram-taxa-de-mortalidade-mais-alta.htm

18. Lalwani P, Salgado BB, Pereira Filho IV, Silva DSS, Morais TBN, Jordão MF, et al. SARS-CoV-2 seroprevalence and associated factors in Manaus, Brazil: baseline results from the DETECTCoV-19 cohort study. Int J Infect Dis 2021;110:141-50. http://doi. org/10.1016/j.ijid.2021.07.017

19. Daumas RP, Azevedo e Silva G, Tasca R, Leite IC, Brasil P, Greco DB, et al. The role of primary care in the Brazilian healthcare system: limits and possibilities for fighting COVID-19. Cad Saude Publica 2020;36(6):e00104120. http://doi. org/10.1590/0102-311X00104120

20. World Health Organization. The rational use of drugs: report of the conference of experts. Nairóbi, 1985 Jul 25-29. Genebra: World Health Organization; 1987.

21. Sackell DL, Rosenberg WM, Gray JA, Haynes RB, Richardson WS. Evidence based medicine: what it is and what it isn't. BMJ 1996;312(7023):71-2. http://doi.org/10.1136/bmj.312.7023.71

22. Sociedade Brasileira de Medicina de Família e Comunidade. Recomendações da SBMFC para a APS durante a pandemia de COVID-19. $3^{a}$ ed. [Internet] 2020. [acessado em 22 jan 2021]. Disponível em: https://www.sbmfc. org.br/wp-content/uploads/2020/07/Recomendac\%CC\%A7o\%CC\%83es-da-SBMFC-para-a-APS-durante-aPandemia_3versa\%CC\%83o_12_07-1.pdf

23. Falavigna $\bar{M}$, Colpani V, Stein $\mathrm{C}$, Azevedo LCP, Bagattini AM, Brito GV, et al. Diretrizes para o tratamento farmacológico da COVID-19. Consenso da Associação de Medicina Intensiva Brasileira, da Sociedade Brasileira de Infectologia e da Sociedade Brasileira de Pneumologia e Tisiologia. Rev Bras Ter Intensiva 2020;32(2):166-96. https://doi.org/10.5935/0103507X.20200039

24. Sociedade Brasileira de Infectologia. Atualizações e recomendações sobre a COVID-19. [Internet] 2020. [acessado em 22 jan 2021]. Disponível em: https://infectologia.org.br/wp-content/uploads/2020/12/atualizacoes-e-recomendacoes-covid-19.pdf

25. Sociedade Brasileira de Infectologia. Associação Médica Brasileira. Informativo conjunto da Associação Médica Brasileira $(\mathrm{AMB})$ e Sociedade Brasileira de Infectologia (SBI) sobre vacinação e tratamento farmacológico preventivo [Internet] 2021. [acessado em 22 jan 2021]. Disponível em: https://infectologia.org.br/wp-content/uploads/2021/01/informativo-conjunto-daamb-e-sbi-sobre-vacinacao-e-tratamento-farmacologico-preventivo-covid-19.pdf

26. Mota DM, Kuchenbecker RDS. Considerações sobre o uso de evidências científicas em tempos de pandemia: o caso da COVID-19. Visa em Debate 2020;8(2):2-9. https://doi.org/10.22239/2317-269X.01541

27. Tritany RF, Tritany EF. Uso racional de medicamentos para COVID-19 na atenção primária à saúde. Saúde em Redes 2020;6(Supl.2):7-16. https://doi.org/10.18310/2446-48132020v6n2\%20Suplem.3205g537

28. Gavilán-Moral E, Barroso AV, Gracia LJ. Como desprescrever medicamentos. In: Gusso G, Lopes JMC, Dias LC. Tratado de Medicina de Família e Comunidade: princípios, formação e prática. 2a ed. Artmed: Porto Alegre; 2018. p. 828-35.

29. Conselho Regional de Farmácia do Estado de São Paulo. Combate à falsificação e roubo de medicamentos: manual de orientação ao farmacêutico [Internet]. [acessado em 22 jan. 2021]. Disponível em: http://www.crfsp.org.br/documentos/ materiaistecnicos/Combate_Falsificacao_e_Roubo_de_Medicamentos.pdf

30. Santos AKC, Araújo TA, Oliveira FS. Farmacoterapia e cuidados farmacêuticos da gripe e resfriado. Journal of Biology \& Pharmacy and Agricultural Management 2020;16(2):137-55.

31. Peres AC. Menos é mais: como a desprescrição pode ser uma forte aliada para a promoção do uso racional de medicamentos. RADIS Comunicação e Saúde [Internet]. 2020. [acessado em 22 jan 2021]. Disponível em: https://radis.ensp.fiocruz.br/ index.php/home/reportagem/menos-e-mais

32. Garcia LP, Duarte E. Infodemia: excesso de quantidade em detrimento da qualidade das informações sobre a COVID-19. Epidemiol Serv Saude 2020;29(4):e2020186. https://doi.org/10.1590/S1679-49742020000400019

33. Stewart M, Brown JB, Weston WW, McWhinney IR, McWilliam CL, Freeman TR. Medicina centrada na pessoa: transformando o método clínico. $3^{\mathrm{a}}$ ed. Porto Alegre: Artmed; 2017.

34. Lima JVO, Cavalcante GL, Braga NSM, Silva AR, Silva TM, Gomes BP, et al. Potential risk of investigated drugs for the treatment of COVID-19: drugs interactions. Rev Pre Infec e Saúde 2020;6:10829. https://doi.org/10.26694/repis.v6i0.10829

35. Molento MB. Ivermectin against COVID-19: the unprecedented consequences in Latin America. One Health 2021;13:100250. https://doi.org/10.1016/j.onehlt.2021.100250

36. Kloetzel K. O diagnóstico clínico: estratégias e táticas. In: Duncan BB, Schimidt MI, Giugliani ERJ. Medicina ambulatorial: condutas de atenção primária baseadas em evidências. $4^{a}$ ed. Porto Alegre: Artmed; 2013. p. 107-18.

37. Lopes JMC. Registro de saúde orientado por problemas. In: Gusso G, Lopes JMC, Dias LC. Tratado de Medicina de Família e Comunidade: princípios, formação e prática. $2^{a}$ ed. Porto Alegre: Artmed; 2018. p. 346-56. 
38. Getahun H, Smith I, Trivedi K, Paulin S, Balkhy HH. Tackling antimicrobial resistance in the COVID-19 pandemic. Bull World Health Organ 2020;98(7):442-442A. https://doi.org/10.2471/BLT.20.268573

39. Brasil. Ministério da Saúde. Gabinete do Ministro. Portaria $n^{\circ} 2.436$, de 21 de setembro de 2017. Aprova a Política Nacional de Atenção Básica, estabelecendo a revisão de diretrizes para a organização da Atenção Básica, no âmbito do Sistema Único de Saúde (SUS). Diário Oficial da União, Poder Executivo. Brasília, 21 de setembro de 2017. [Internet]. 2017. [acessado em 08 nov 2021]. Disponível em: https://bvsms.saude.gov.br/bvs/saudelegis/gm/2017/prt2436_22_09_2017.html

40. Lopes JMC. Princípios da medicina de família e comunidade. In: Gusso G, Lopes JMC, Dias LC. Tratado de Medicina de Família e Comunidade: princípios, formação e prática. 2a ed. Porto Alegre: Artmed; 2018. p. 1-10.

41. Hurtado RL, Lasmar MC. Medicamentos falsificados e contrabandeados no Brasil: panorama geral e perspectivas de combate ao seu consumo. Cad Saúde Pública 2014;30(4):891-5. https://doi.org/10.1590/0102-311X00107013

42. Brasil. Ministério da Saúde. Agência Nacional de Vigilância Sanitária. Protocolo de segurança na prescrição, uso e administração de medicamentos. Protocolo coordenado pelo Ministério da Saúde e ANVISA em parceria com FIOCRUZ e FHEMIG [Internet] 2013. [acessado em 22 jan 2021]. Disponível em: https://www20.anvisa.gov. br/segurancadopaciente/ index.php/publicacoes/item/seguranca-na-prescricao-uso-e-administracao-de-medicamentos

43. Lucena RCB. A descentralização na vigilância sanitária: trajetória e descompasso. Rev Adm Pública 2015;49(5):1107-20. https://doi.org/10.1590/0034-7612137128

44. Fick DM, Cooper JW, Wade WE, Waller JL, Maclean JR, Beers MH. Updating the Beers criteria for potentially inappropriate medication use in older adults: results of a US consensus panel of experts. Arch Intern Med 2003;163(22):2716-24. https:// doi.org/10.1001/archinte.163.22.2716

45. Silva LMC, Araújo JL. Clinical and community pharmacist's role in the COVID-19 pandemic. Research, Society and Development 2020;9(7):e684974856. https://doi.org/10.33448/rsd-v9i7.4856

46. Tabakman R. A notícia é outra quando se trata da descoberta de novos medicamentos. Observatório da Imprensa [Internet] 2018. [acessado em 22 jan 2021]. Disponível em: http://www.observatoriodaimprensa.com.br/imprensa-e-saude/a-noticia-eoutra-quando-se-trata-da-descoberta-de-novos-medicamentos/

47. Máximo W. Área técnica da Anvisa recomenda uso emergencial da CoronaVac. Agência Brasil de 17 de janeiro de 2021 [Internet]. [acessado em 22 jan 2021]. Disponível em: https://agenciabrasil.ebc.com.br/saude/noticia/2021-01/area-tecnicada-anvisa-recomenda-uso-emergencial-da-coronavac 